\title{
Inertial-range spectrum of whistler turbulence
}

\author{
Y. Narita ${ }^{1}$ and S. P. Gary ${ }^{2}$ \\ ${ }^{1}$ Institut für Geophysik und extraterrestrische Physik, Technische Universität Braunschweig, Mendelssohnstr. 3, \\ 38106 Braunschweig, Germany \\ ${ }^{2}$ Los Alamos National Laboratory, Los Alamos, New Mexico 87545, USA
}

Received: 24 December 2009 - Revised: 16 February 2010 - Accepted: 17 February 2010 - Published: 19 February 2010

\begin{abstract}
We develop a theoretical model of an inertialrange energy spectrum for homogeneous whistler turbulence. The theory is a generalization of the Iroshnikov-Kraichnan concept of the inertial-range magnetohydrodynamic turbulence. In the model the dispersion relation is used to derive scaling laws for whistler waves at highly oblique propagation with respect to the mean magnetic field. The model predicts an energy spectrum for such whistler waves with a spectral index -2.5 in the perpendicular component of the wave vector and thus provides an interpretation about recent discoveries of the second inertial-range of magnetic energy spectra at high frequencies in the solar wind.
\end{abstract}

Keywords. Interplanetary physics (Plasma waves and turbulence) - Space plasma physics (Turbulence; Wave-wave interactions)

\section{Introduction}

Whistler turbulence is widely observed not only in the Earth's magnetosphere (Santolik and Gurnett, 2002) but also in planetary plasma environments such as Venus (Scarf et al., 1980), Jupiter (Gurnett et al., 1979), Neptune (Gurnett et al., 1990) and in the solar wind (Lengyel-Frey et al., 1996). At relatively low frequencies, inertial-range turbulence in the solar wind typically exhibits magnetic field fluctuation spectra which scale as frequency to the $-5 / 3$ power, but beyond a spectral breakpoint near $0.2-0.5 \mathrm{~Hz}$ such spectra become steeper at higher frequencies (Leamon et al., 1998; Sahraoui et al., 2009). On small scales (scales shorter than the proton gyro-radius or inertial length) the one-fluid approximation of magnetohydrodynamics (MHD) is no longer valid and the behavior of the plasma and electromagnetic fields should be

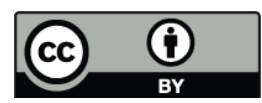

Correspondence to: $\mathrm{Y}$. Narita

(y.narita@tu-bs.de) described by an electron-magnetohydrodynamics (EMHD) model, or preferably via a kinetic treatment. In this highfrequency, short-wavelength regime, two hypotheses have been put forward to describe the modes which may constitute this turbulence. One school of thought advocates kinetic Alfvén waves at frequencies below the proton cyclotron frequency as the fundamental mode of this regime (Leamon et al., 1998; Bale et al., 2005; Sahraoui et al., 2009). Another point of view is that whistler fluctuations at frequencies above the proton cyclotron frequency are the more important constituent of this regime (Stawicki et al., 2001). Taking the latter point of view, Saito et al. (2008) presented particle-incell (PIC) simulations showing whistler turbulence tends to transport fluctuation energy toward the perpendicular direction to the mean magnetic field.

Here we develop a theoretical model of whistler turbulence which is a generalization of scaling laws for magnetohydrodynamic turbulence proposed by Iroshnikov (1964) and Kraichnan (1965). While the theory of MHD turbulence is based on interactions of counter-propagating Alfvén waves, our model deals with interactions of obliquely propagating whistler waves, considering separately the cases of wave-wave interactions due to propagation components both quasi-parallel and quasi-perpendicular to the mean magnetic field. Assumptions we use are: (1) there is no wave damping; (2) waves follow the cold plasma whistler dispersion relation; (3) interaction is weak or incoherent such that many wavewave collisions are needed to transfer energy from one scale to another. Furthermore, we follow recent solar wind observations (Alexandrova et al., 2009) and PIC simulation studies (Saito et al., 2008) and EMHD computations (Biskamp et al., 1996; Dastgeer et al., 2000; Cho and Lazarian, 2004, 2009) and assume (4) turbulent whistler fluctuations propagate predominantly in directions quasi-perpendicular to the background magnetic field. Our theory predicts a fluctuating magnetic energy spectrum which scales as $k_{\perp}^{-5 / 2}$, consistent with the slopes of high frequency spectra observed in 
the solar wind (Alexandrova et al., 2009; Kiyani et al., 2009; Sahraoui et al., 2009).

A different model of whistler turbulence has been developed by Krishan and Mahajan (2004) using Hall-MHD theory. This theory predicts that the magnetic energy spectrum at wavelengths shorter than the breakpoint of the MHD inertial range should scale as $k^{-11 / 3}$ or $k^{-13 / 3}$, much steeper than our prediction or the recent solar wind observations cited above. The reason for this discrepancy may lie in the difference between Hall-MHD, which applies to wavelengths of order ion inertial or ion gyroradius scales, and our theory which applies to relatively shorter wavelengths where dimensions scale with the electron, rather than the ion, mass.

\section{Theory}

As in the Iroshnikov-Kraichnan theory for MHD turbulence, there are two fundamental time scales in whistler turbulence. One is the eddy turnover time around the mean magnetic field

$\tau_{\mathrm{ed}}=\frac{l}{v}$,

and the other is the whistler-interaction time

$\tau_{\mathrm{w}}=\frac{l}{v_{\mathrm{g}}}$,

where the length $l$ and the group speed $v_{\mathrm{g}}$ are dependent on the propagation directions (denoted by the subscripts $\perp$ and $\|)$. Whistler waves are dispersive and follow at $k c / \omega_{\mathrm{e}} \ll$ 1 the cold dispersion relation, which can approximately be written as

$\frac{\omega}{\Omega_{\mathrm{e}}}=\frac{k_{\|} k c^{2}}{\omega_{\mathrm{e}}^{2}}$

where $\Omega_{\mathrm{e}}, \omega_{\mathrm{e}}$ and $c$ denote the cyclotron frequency and the plasma frequency for electrons, and the speed of light, respectively. We use the expression $k=|k|=\sqrt{k_{\|}^{2}+k_{\perp}^{2}}$. For waves propagating perpendicular and parallel to the mean magnetic field the group velocity is

$\left(\begin{array}{c}v_{\mathrm{g} \perp} \\ v_{\mathrm{g} \|}\end{array}\right)=\left(\begin{array}{c}\frac{\Omega_{\mathrm{e}} c^{2}}{\omega_{\mathrm{e}}^{2}} \frac{k_{\|} k_{\perp}}{k} \\ \frac{\Omega_{\mathrm{e}} c^{2}}{\omega_{\mathrm{e}}^{2}}\left(k+\frac{k_{\|}^{2}}{k}\right)\end{array}\right)$.

We estimate the energy transfer time (or the cascade time) as

$$
\tau_{\text {cas }}=M \tau_{\mathrm{w}},
$$

which means that we need to estimate the number of wavewave collisions, $M$, to transfer energy from one scale to another. Consider a fraction of energy transported by one wave packet interaction

$\frac{\Delta E}{E}=\frac{\Delta v^{2}}{v^{2}} \sim \frac{\Delta v}{v} \ll 1$, where $E$ is the total amount of energy on one scale, $\Delta E$ is the amount of energy that is transported to the other scale by eddy distortion and is associated with the velocity change $\Delta v$. The whistler interaction time is much smaller than that of eddy distortion, and therefore $\Delta E / E$ and $\Delta v / v$ are very small.

If the wave interaction occurs coherently (one after another immediately) then we need $N$ wave collisions to complete the energy transport to the next scale with

$N=\frac{\tau_{\mathrm{ed}}}{\tau_{\mathrm{w}}}$.

In this case the energy transfer time restores the eddy turnover time $\tau_{\text {cas }}=\tau_{\text {ed }}$ (substituting $M$ by $N$ in Eq. 5) and turbulence is hydrodynamic-like (Kolmogorov scaling) in the sense that the energy transport is predominantly made by eddy distortion.

If the whistler wave interaction occurs incoherently, that is the interaction occurs only occasionally or is ineffective such that a large number of collisions are needed for the wave interaction to complete the energy transport from scale to scale, we estimate the transfer time by substituting $M$ by $N^{2}$ (which is one of the assumptions in the Iroshnikov-Kraichnan model to use the smallest number for wave-wave interactions for a fast energy cascade process) and obtain

$\tau_{\text {cas }}=N^{2} \tau_{\mathrm{w}}$.

In this case $\tau_{\text {cas }}$ is expressed as a combination of $\tau_{\mathrm{ed}}$ and $\tau_{\mathrm{w}}$ (Cho and Lazarian, 2004),

$\tau_{\mathrm{cas}}=\frac{\tau_{\mathrm{ed}}^{2}}{\tau_{\mathrm{w}}}$.

We use the incoherent case in the following discussion.

The electron velocity $\boldsymbol{v}_{\mathrm{e}}$ is estimated by the expression of electric current in EMHD,

$e n_{\mathrm{e}} \boldsymbol{v}_{\mathrm{e}}=\frac{1}{\mu_{0}} \nabla \times \boldsymbol{B}$.

On the assumption that $\boldsymbol{v}_{\mathrm{e}} \simeq \delta \boldsymbol{v}_{\mathrm{e}}$ (no bulk velocity),

$\left|\delta \boldsymbol{v}_{\mathrm{e}}\right|=\frac{\Omega_{\mathrm{e}} c^{2}}{\omega_{\mathrm{e}}^{2}}\left|\frac{\delta B}{B_{0}}\right| k$.

Then the eddy turnover time is estimated for EMHD (assuming ions are in rest due to large inertia),

$\tau_{\mathrm{ed}}=\frac{l}{\left|\boldsymbol{v}_{\mathrm{e}}\right|}=\frac{\omega_{\mathrm{e}}^{2}}{\Omega_{\mathrm{e}} c^{2}}\left|\frac{B_{0}}{\delta B}\right| \frac{1}{k^{2}}$,

Now define the energy transfer rate using $\tau_{\text {cas }}$ as

$$
\begin{aligned}
\epsilon & =\frac{1}{\tau_{\text {cas }}}\left(\left|\delta \boldsymbol{v}_{\mathrm{e}}\right|^{2}+\frac{|\delta B|^{2}}{\mu_{0} \rho_{\mathrm{e}}}\right) \\
& \simeq \frac{|\delta B|^{2}}{\tau_{\text {cas }} \mu_{0} \rho_{\mathrm{e}}} .
\end{aligned}
$$


Note that the contribution of the kinetic energy is dropped as it is small at $k^{2} c^{2} / \omega_{\mathrm{e}}^{2} \ll 1$ (Eq. 11). While the energy transfer time $\tau_{\text {cas }}$ represents the time scale on which the energy cascade from one scale to another is completed by $N^{2}$ wave collisions and has the units of time, the energy transfer rate $\epsilon$ represents the amount of transferred energy within $\tau_{\text {cas }}$ and is given in units of energy (or squared velocity) divided by time. Both $\tau_{\text {cas }}$ and $\epsilon$ are dependent on the picture of wave-wave interaction, in particular in which component of the group velocity the interaction occurs. In the following discussion we assume constancy of the energy transfer rate, $\epsilon=$ const., and quasi-perpendicular propagation, $k_{\|} \ll k_{\perp}$. But we consider two distinct cases: the quasi-perpendicular part of the interaction, and the quasi-parallel part of the interaction.

\subsection{Quasi-perpendicular interaction}

For waves interacting primarily through the $k_{\perp}$ component of the wave vector, the whistler interaction time is

$\tau_{\mathrm{w} \perp}=\frac{l_{\perp}}{v_{\mathrm{g} \perp}}=\frac{\omega_{\mathrm{e}}^{2}}{\Omega_{\mathrm{e}} c^{2}} \frac{k}{k_{\|} k_{\perp}^{2}}$,

which yields according to Eq. (9) the energy transfer time

$\tau_{\text {cas } \perp}=\frac{\omega_{\mathrm{e}}^{2}}{\Omega_{\mathrm{e}} c^{2}}\left|\frac{B_{0}}{\delta B}\right|^{2} \frac{k_{\|} k_{\perp}^{2}}{k^{5}}$

and the energy transfer rate

$\epsilon_{\perp}=\frac{\Omega_{\mathrm{e}} c^{2}}{\omega_{\mathrm{e}}^{2}} \frac{|\delta B|^{4}}{B_{0}^{2} \mu_{0} \rho_{\mathrm{e}}} \frac{k^{5}}{k_{\|} k_{\perp}^{2}}$.

The assumption that $\epsilon_{\perp}=$ const. allows one to express the magnetic field fluctuation as

$|\delta B|^{4}=\epsilon_{\perp} \frac{\omega_{\mathrm{e}}^{2} B_{0}^{2} \mu_{0} \rho_{\mathrm{e}}}{\Omega_{\mathrm{e}} c^{2}} \frac{k_{\|} k_{\perp}^{2}}{k^{5}}$.

For quasi-perpendicular propagation we have $k \simeq k_{\perp}$. Using the relation $\Delta k_{\perp} \propto k_{\perp}$, we obtain the energy spectrum as

$$
\begin{aligned}
E\left(k_{\perp}\right) & \equiv \frac{1}{\Delta k_{\perp}} \frac{|\delta B|^{2}}{\mu_{0} \rho_{\mathrm{e}}} \\
& \simeq\left(\epsilon_{\perp} \omega_{\mathrm{e}} \Omega_{\mathrm{e}}\right)^{1 / 2}\left\langle k_{\|}\right\rangle^{1 / 2} k_{\perp}^{-5 / 2} .
\end{aligned}
$$

The spectral slope is -2.5 in the perpendicular direction which is steeper than MHD turbulence spectrum and close to the index obtained in the high-frequency spectra of the solar wind (Alexandrova et al., 2009; Kiyani et al., 2009; Sahraoui et al., 2009). $\left\langle k_{\|}\right\rangle$denotes the average parallel wave number representing the half-value-width of energy spectrum in the $k_{\|}$direction. The positive power of $k_{\|}$in Eq. (20) which suggests an increasing energy with increasing parallel wave vector is not of concern. This equation is valid only at $k_{\|} \ll k_{\perp}$ and may not be extrapolated to the regime $k_{\|} \sim k_{\perp}$. It is interesting to note that Eq. (20) reproduces the energy spectrum of EMHD turbulence, $E\left(k_{\perp}\right) \propto k_{\perp}^{-7 / 3}$ when the condition of whistler critical balance (Cho and Lazarian, 2004), $k_{\|} \sim k_{\perp}^{1 / 3}$, is used. In this sense our scaling law may be regarded as a generalization of the EMHD turbulence model.

\subsection{Quasi-parallel interaction}

If whistler waves at quasi-perpendicular propagation are nevertheless interacting primarily through wave vector components quasi-parallel to the background magnetic field, the time scale for such interactions is determined by the parallel group velocity

$\tau_{\mathrm{w} \|}=\frac{l_{\|}}{v_{\mathrm{g} \|}}=\frac{\omega_{\mathrm{e}}^{2}}{\Omega_{\mathrm{e}} c^{2}} \frac{1}{k_{\|} k}$,

which reduces to $\tau_{\mathrm{w} \perp}$ under approximation $k \sim k_{\perp} \gg k_{\|}$, therefore the scaling laws and the spectrum become the same as that for parallel wave interaction. In more detail, the cascade time is given as

$\tau_{\text {cas } \|}=\frac{\omega_{\mathrm{e}}^{2}}{\Omega_{\mathrm{e}} c^{2}}\left|\frac{B_{0}}{\delta B}\right|^{2} \frac{k_{\|}}{k^{3}}$

and the energy transfer rate is

$\epsilon_{\|}=\frac{\Omega_{\mathrm{e}} c^{2}}{\omega_{\mathrm{e}}^{2}} \frac{|\delta B|^{4}}{B_{0}^{2} \mu_{0} \rho_{\mathrm{e}}} \frac{k^{3}}{k_{\|}}$.

Again, the assumption that $\epsilon_{\|}=$(const.) gives the expression for the magnetic field fluctuation as

$|\delta B|^{4}=\epsilon_{\|} \frac{\omega_{\mathrm{e}}^{2} B_{0}^{2} \mu_{0} \rho_{\mathrm{e}}}{\Omega_{\mathrm{e}} c^{2}} \frac{k_{\|}}{k^{3}}$.

For highly obliquely propagating waves, $k \sim k_{\perp}$, this scaling has the same form as that for the quasi-perpendicular wave interaction, and we obtain the energy spectrum in the perpendicular wave number domain as

$E\left(k_{\perp}\right) \simeq\left(\epsilon_{\|} \omega_{\mathrm{e}} \Omega_{\mathrm{e}}\right)^{1 / 2}\left\langle k_{\|}\right\rangle^{1 / 2} k_{\perp}^{-5 / 2}$,

which has the same form as Eq. (20) except for the difference between $\epsilon_{\perp}$ and $\epsilon_{\|}$. It is interesting that the both interaction types (wave-wave scattering in the parallel and perpendular fluctuation components) yield the same slope in the spectrum.

\section{Conclusions}

In this paper we have generalized the Iroshnikov-Kraichnan concept of magnetohydrodynamic turbulence to apply it to homogeneous whistler turbulence at propagation highly oblique to the mean magnetic field. The model predicts an energy spectrum for such turbulence with a spectral index of -2.5 in the perpendicular component of the wave vector and is consistent with measurements of high-frequency spectral indices in the solar wind. Although our derivation is suggestive, there are many further issues which must be addressed with respect to whistler turbulence. 
1. We have assumed that waves are propagating at highly oblique direction, since our work is motivated by the results obtained in the solar wind observations and simulations. The cause of anisotropy of large propagation angles is an interesting and very important question. Perhaps it is determined by wave interaction process such as three or four wave couplings, or it is determined by anisotropic decay of wave amplitudes due to cyclotron or Landau damping.

2. The inclusion of wave damping would therefore be an important addition to this model; the linear theory dissipation calculations of Gary et al. (2009) for fluctuations in electron-positron plasmas may be instructive in this regard.

3. Polarization of whistler waves, in particular the ratio of the parallel and perpendicular components of the fluctuating magnetic field, could also be incorporated in our model.

4. The particle-in-cell simulations of Saito et al. (2008) suggest that the spectral index of two-dimensional whistler turbulence at quasi-perpendicular propagation is larger (steeper spectra) than the value of -2.5 derived here. Further PIC simulations of whistler turbulence would be useful to help determine whether such steep spectra are due to Landau damping or other kinetic effects.

5. Group velocity of whistler waves changes significantly in dependence upon the propagation angle, with the wave being purely electromagnetic for parallel and increasingly electrostatic for oblique propagation. Effects of different propagation angles (from quasi-parallel, to oblique, and to quasi-perpendicular directions from the mean magnetic field) should be investigated systematically.

6. The Hall-MHD theory of Krishan and Mahajan (2004) predicts that whistler magnetic spectra should scale as $k^{-11 / 3}$ and then as $k^{-13 / 3}$ at very short wavelengths. This appears to be much steeper than recent solar wind observations, so it is imoprtant to distinguish between Hall-MHD and whistler turbulence in simulations and observations.

Acknowledgements. This work is supported by the program "Solar wind turbulence at short wavelengths" of the National Aeronautics and Space Administration. The work of YN was also supported by Budesministerium für Wirtschaft und Technologie and Deutsches Zentrum für Luft- und Raumfahrt, Germany, under contract 50 OC 0901.

Topical Editor I. A. Daglis thanks one anonymous referee for her/his help in evaluating this paper.

\section{References}

Alexandrova, O., Saur, J., Lacombe, C., Mangeney, A., Mitchell, J., Schwartz, S. J., and Robert, P.: Universality of Solar-wind turbulent spectrum from MHD to electron scales, Phys. Rev. Lett., 103, 165003, doi:10.1103/PhysRevLett.103.165003, 2009.

Bale, S. D., Kellogg, P. J., Mozer, F. S., Horbury, T. S., and Rème, H.: Measurement of the electric fluctuation spectrum of magnetohydrodynamic turbulence, Phys. Rev. Lett., 94, 215002, doi:10.1103/PhysRevLett.94.215002, 2005.

Biskamp, D., Schwarz, E., and Drake, J. F.: Two-dimensional electron magnetohydrodynamic turbulence, Phys. Rev. Lett., 76, 1264-1267, 1996.

Cho, J. and Lazarian, A.: The anisotropy of electron magnetohydrodynamic turbulence, Astrophys. J., 615, L41-L44, 2004.

Cho, J. and Lazarian, A.: Simulations of electron magnetohydrodynamic turbulence, Astrophys. J., 701, 236-252, 2009.

Dastgeer, S., Das, A., Kaw, P., and Diamond, P. H.: Whistlerization and anisotropy in two-dimensional electron magnetohydrodynamic turbulence, Phys. Plasmas, 7, 571-579, 2000.

Gary, S. P., Karimabadi, H., and Roytershteyn, V. S.: Dissipation wavenumbers for turbulence in electron-positron plasmas, Astrophys. J., 701, 1695-1700, 2009.

Gurnett, D. A., Shaw, R. R., Anderson, R. R., and Kurth, W. S.: Whistlers observed by Voyager 1 - Detection of lightning on Jupiter, Geophys. Res. Lett., 6, 511-514, 1979.

Gurnett, D. A., Kurth, W. S., Cairns, I. H., and Granroth, L. J.: Whistlers in Neptune's magnetosphere - Evidence of atmospheric lightning, J. Geophys. Res., 95, 20967-20976, 1990.

Iroshnikov, P. S.: Turbulence on a conducting fluid in a strong magnetic field, Sov. Astron., 7, 566-571, 1964.

Kiyani, K. H., Chapman, S. C., Khotyanintsev, Y. V., Dunlop, M. W., and Sahraoui, F.: Global scale-invariant dissipation in collisionless plasma turbulence, Phys. Rev. Lett., 103, 075006, doi:10.1103/PhysRevLett.103.075006, 2009.

Kraichnan, R. H.: Inertial-range spectrum of hydromagnetic turbulence, Phys. Fluids, 8, 1385-1387, 1965.

Krishan, V. and Mahajan, S. M.: Magnetic fluctuations and Hal magnetohydrodynamic turbulence in the solar wind, J. Geophys. Res., 109, A11105, doi:10.1029/2004JA010496, 2004.

Lengyel-Frey, D., Hess, R. A., MacDowall, R. J., Stone, R. G., Lin, N., Balogh, A., and Forsyth, R.: Ulysses observations of whistler waves at interplanetary shocks and in the solar wind, J. Geophys. Res., 101, 27555-27564, 1996.

Leamon, R. J., Smith. C. W., Ness, N. F., Matthaeus, W. H., and Wong, H. K.: Observational constraints on the dynamics of the interplanetary magnetic field dissipation range, J. Geophys. Res., 103, 4775-4787, 1998.

Santolik, O. and Gurnett, D. A.: Propagation of auroral hiss at high altitudes, Geophys. Res. Lett., 29, 1481, doi:10.1029/2001GL013666, 2002.

Sahraoui, F., Goldstein, M. L., Robert, P., and Khotyaintsev, Y. V.: Evidence of a cascade and dissipation of solar-wind turbulence at the electron gyroscale Phys. Rev. Lett., 102, 231102, doi:10.1103/PhysRevLett.102.231102, 2009.

Saito, S., Gary, S. P., Li, H., and Narita, Y.: Whistler turbulence: Particle-in-cell simulations, Phys. Plasmas, 15, 102305, doi:10.1063/1.2997339, 2008. 
Scarf, F. L., Taylor, W. W. L., Russell, C. T., and Brace, L. H.: Lightning on Venus - Orbiter detection of whistler signals, J. Geophys. Res., 85, 8158-8166, 1980.
Stawicki, O., Gary, S. P., and Li, H.: Solar wind magnetic fluctuation spectra: Dispersion versus damping, J. Geophys. Res., 106, 8273-8282, 2001. 